\title{
Semiclassical Calculations of Vibrational Energy Levels for Non- Separable Systems Using the Birkhoff-Gustavson Normal Form
}

\author{
Randall T. Swimm \\ William \& Mary \\ John B. Delos \\ William \& Mary, jbdelos@wm.edu
}

Follow this and additional works at: https://scholarworks.wm.edu/aspubs

Part of the Physics Commons

\section{Recommended Citation}

Swimm, Randall T. and Delos, John B., Semiclassical Calculations of Vibrational Energy Levels for NonSeparable Systems Using the Birkhoff-Gustavson Normal Form (1979). Journal of Chemical Physics, 71(4), 1706-1717.

https://doi.org/10.1063/1.438521

This Article is brought to you for free and open access by the Arts and Sciences at W\&M ScholarWorks. It has been accepted for inclusion in Arts \& Sciences Articles by an authorized administrator of W\&M ScholarWorks. For more information, please contact scholarworks@wm.edu. 


\title{
Semiclassical calculations of vibrational energy levels for nonseparable systems using the Birkhoff-Gustavson normal form
}

\author{
R. T. Swimm and J. B. Delos \\ Department of Physics, College of William and Mary, Williamsburg, Virginia 23185 \\ (Received 19 February 1979; accepted 23 April 1979)
}

\begin{abstract}
We present a semiclassical method of calculating vibrational energy levels for a system of nonseparable coupled oscillators. For a Hamiltonian written as a power series in which the leading terms are given by a sum of one-dimensional harmonic oscillator Hamiltonians, the method involves transforming the original classical Hamiltonian via a succession of canonical transformations into a normal form which is a power series originally defined by Birkhoff and later generalized by Gustavson. Two cases are distinguished. If the harmonic oscillator frequencies in the unperturbed Hamiltonian are incommensurable, then the normal form is a power series whose terms are products of one-dimensional harmonic oscillator Hamiltonians; if the frequencies in the unperturbed Hamiltonian are commensurable, then additional terms which cannot be written as products of one-dimensional harmonic oscillator Hamiltonians enter into the normal form. Once the normal form is obtained, semiclassical quantization of action variables is straightforward. The incommensurable case yields a formula for the energy spectrum which is a power series in the quantum numbers. The commensurable case is more complicated, and yields a form from which energy levels may be obtained individually by numerical calculation and quantization of a one-dimensional phase integral. Nonseparable two-dimensional examples are treated for each case. The results obtained for both cases show excellent agreement with quantum mechanical calculations. The quantum calculations indicate that all of the energy levels fall into a regular pattern.
\end{abstract}

\section{INTRODUCTION}

The work to be described in this paper has two goals. The first is to develop a new mostly analytical semiclassical method for calculation of vibrational energy levels of nonseparable systems of coupled oscillators. The second is to use this method to study and interpret the quantum-mechanical energy spectrum for such systems. In particular, we search for evidence of an "irregular" spectrum.

It is useful to summarize some of the rather surprising properties of classical mechanical systems that have been established in the past 15 years. It is well known ${ }^{1}$ that, for any system of coupled oscillators, so long as the forces are linear functions of the displacements, the standard normal-mode analysis separates the variables, and shows that the general motion is a superposition of independent harmonic oscillations. More generally, all separable, bound classical systems with $n$ degrees of freedom have a set of related properties: (i) the system possesses at least $n$ isolating integrals ${ }^{2}$ of the motion (an integral is isolating if it reduces by one the dimension of the set of phase space points which the trajectory may approach); (ii) the motion is multiply periodic (quasiperiodic) and topologically equivalent to flow on an $n$-dimensional torus in the $2 n$-dimensional phase space; (iii) the motion can be described in terms of action-angle variables; (iv) the Hamilton-Jacobi equation possesses a complete integral which generates the transformation to action-angle variables. All of these properties are established in the standard textbooks on classical mechanics, ${ }^{1}$ and such motion is said to be "regular."

Systems for which the Hamilton-Jacobi equation is not separable, however, may possess fewer than $n$ isolating integrals. Consequently, while some trajectories are still multiply periodic, other irregular trajectories exist for which the motion is not multiply periodic, the trajectory is not confined to an $n$-dimensional torus, there is no complete integral for the Hamilton-Jacobi equation having the properties needed to generate a transformation to action-angle variables, and actionangle variables do not exist.

The full complexity of such classical systems is revealed in the work of Kolmogorov, ${ }^{3}$ Arnold, ${ }^{4}$ and Moser $^{5}$ (KAM), which has been made accessible to nonmathematicians by Ford. ${ }^{6}$ KAM consider systems for which the Hamiltonian consists of an unperturbed part $H_{0}$, having only multiply periodic trajectories, and a general analytic perturbation $H_{1}$. They prove that most of the regular trajectories of the unperturbed system remain regular under sufficiently small perturbations. There may be a set of irregular trajectories (and this set may even be dense), but the regular trajectories outweigh them in somewhat the same way that irrational numbers outweigh rational numbers on the real line. ${ }^{7}$ As the size of the perturbation term increases, the regions of irregular motion increase, and they may eventually fill practically all of the energy shell in phase space. $\mathrm{Nu}-$ merical experiments displaying this behavior were made by Henon and Heiles, ${ }^{8}$ Ford, ${ }^{6}$ and others.

The existence of these two types of motion in classical mechanics suggests that there may be two distinct types of behavior in quantum mechanics. The set of quantum states which correspond via the semiclassical approximation to regular classical trajectories may be called the regular part of the spectrum. ${ }^{9}$ For these states, the function $S$ which is a proper solution to the Hamilton-Jacobi equation would correspond to the phase of the wave function, and the energy spectrum could be calculated approximately by a generalized Bohr-Som- 
merfeld procedure. In any such procedure, the possible presence of a (presumably small) set of irregular trajectories is ignored; the Hamiltonian is transformed canonically to a function of the actions, and the quantization of action variables gives the energy spectrum. In this way, a set of $n$ quantum numbers is assigned to each quantum state.

There now exist several numerical methods for carrying out such calculations. ${ }^{10-14}$ In the next section, a new method is described which allows the solution to the classical problem to be carried out analytically. ${ }^{15}$ By using a kind of perturbation theory in classical mechanics, a power series Hamiltonian can be reduced to a certain "normal form," which (in the simplest case) is directly related to a power series in the action variables. The quantum spectrum then follows trivially.

What is the behavior of quantum states for which the corresponding classical trajectories are irregular? Percival ${ }^{9}$ has suggested that there will be no unambiguous assignment of $n$ quantum numbers to each of these states, that the energies of these states may be especially sensitive to small changes in the Hamiltonian, and that the distribution of energy levels could appear to be random.

In Sec. III of this paper, the method developed in Sec. II is applied to the Henon-Heiles Hamiltonian, which is known to exhibit a transition between dominantly regular and dominantly irregular classical trajectories. Three results are found: (i) the normal form very accurately describes the lower energy levels of this system; (ii) as the energy increases the error in the (truncated) normal form also increases until, in the case considered, the numerical results are not sufficiently accurate to make an unambiguous correlation between semiclassical calculations and exact quantum calculations; however, (iii) the pattern established in the lower quantum levels persists up to the dissociation energy - there is no sign of irregular or "random" spacings of energy levels, and it appears that every bound state can be characterized by two quantum numbers.

\section{THE BIRKHOFF-GUSTAVSON NORMAL FORM}

In this section, we describe a method of treating nonseparable classical systems that was originally developed by Birkhoff ${ }^{16}$ and later extended by Gustavson. ${ }^{17}$ Birkhoff's method provides a procedure to transform the original nonseparable oscillator Hamiltonian canonically into the normal form consisting of a power series in one-dimensional harmonic oscillator Hamiltonians. Because of the particular simplicity of this form, the Hamiltonian can be easily quantized.

The result obtained by Birkhoff is that, given a Hamiltonian $H$ which can be written as a formal power series without constant or linear terms, and such that the quadratic terms may be written as a sum of uncoupled harmonic oscillator terms with incommensurable frequencies, there exists a formal real canonical transformation generated by a power series such that $H$ is transformed into a Hamiltonian which is a power series in one-dimensional uncoupled harmonic oscillator Hamiltonians, i.e.,

$$
H \rightarrow \Gamma\left(\xi_{1}^{2}+\eta_{1}^{2}, \ldots, \xi_{\pi}^{2}+\eta_{n}^{2}\right),
$$

where $\xi_{k}$ are canonical coordinates, $\eta_{k}$ are canonical momenta, and $\Gamma$ is a formal power series in $\xi_{k}^{2}+\eta_{k}^{2}$. If the series $\Gamma$ were to converge, the new equation could be easily integrated, the transformation to angle-action variables would be straightforward, $n$ independent isolating integrals would exist, and the motion would be multiply periodic.

Unfortunately, Siegel ${ }^{18,5}$ has shown that Birkhoff's normal form diverges in general because of small divisors in the coefficients. In hindsight, it is easy to see why this must be so: The normal form cannot describe irregular motion, but irregular trajectories are known to exist, and they may be dense; hence, the radius of convergence of the normal form must be zero.

Birkhoff's method was applied by Gustavson in order to obtain power series expressions for isolating integrals, and to analytically predict the Poincaré surfaces of section for the Henon and Heiles system. (Since the potential treated had commensurable frequencies, Gustavson had to modify Birkhoff's method somewhat.) These calculations showed that, despite the known divergence of the power series representing the normal form, a truncated series can give accurate approximations to the regular trajectories (but not, of course, to the irregular trajectories).

The procedure for transforming to Birkhoff's normal form is reviewed here, although it is an essentially unmodified form of the procedure presented in Gustavson's paper. ${ }^{17}$

Consider a system described by a Hamiltonian

$$
H(u, v)=H^{(2)}(u, v)+H^{(3)}(u, v)+\cdots,
$$

which is a power series in coordinates $u$ and momenta $v$. $H^{(s)}$ is taken to be a homogeneous polynomial of degree $s$ :

$$
H^{(s)}(u, v)=\sum_{\substack{i+j=s \\ i, j \neq 0}} a_{1,} u^{i} v^{j}, \quad s=2,3, \ldots
$$

For systems in which $H^{(2)}$ is positive definite, there exists a canonical transformation $(u, v)-(q, p)$ which transforms $H^{(2)}$ into the form

$$
H^{(2)}(q, p)=\sum_{k=1}^{n} \frac{\omega_{k}}{2}\left(q_{k}^{2}+p_{k}^{2}\right)
$$

Following Gustavson, we now define a normal form. Let $H(q, p)$ be a Hamiltonian with $H^{(2)}(q, p)$ as given in Eq. (2). Then we say that $H(q, p)$ is in normal form if $D H(q, p)=0$, where

$$
D=-\sum_{k=1}^{n} \omega_{k}\left(q_{k} \frac{\partial}{\partial p_{k}}-p_{k} \frac{\partial}{\partial q_{k}}\right) .
$$

This is equivalent to requiring that the Poisson bracket of $H^{(2)}$ with $H$ vanish, since $D$ is given by

$$
D=-\left[H^{(2)},\right] \text {. }
$$

(The minus sign is included here to be consistent with the quantity, Gustavson calls $D$; apparently his Eq. (2.4) should also have this minus sign.) 
A power series Hamiltonian can be transformed to normal form by a sequence of canonical transformations. Each transformation is defined by means of a type-2 generating function $F_{2}$ such that

$$
F_{2}(P, q)=\sum_{k} P_{k} q_{k}+W^{(s)}(P, q)
$$

where $P$ are the new canonical momenta and where the properties of $W^{(s)}$ will be described presently. (Where the abbreviated notation $P, Q, p$, or $q$ is used, $P$ means $P_{1}, \ldots, P_{n}$, etc.) The resulting transformation equations are

$$
\begin{aligned}
& Q=q+\frac{\partial W^{(s)}}{\partial P}, \\
& p=P+\frac{\partial W^{(s)}}{\partial q}, \\
& H(\phi, q)=\Gamma(P, Q),
\end{aligned}
$$

where $Q$ is the coordinate canonically conjugate to $P$, and where $\Gamma$ is the Hamiltonian in the new variables. Beginning with $s=3$, the transformations sequentially normalize the terms of degree $s$ in the Hamiltonian. This can be achieved by taking $W^{(s)}$ to be a homogeneous polynomial of degree $s$.

Substituting Eqs. (6a) and (6b) into (6c), we obtain

$$
H\left(P+\frac{\partial W^{(s)}}{\partial q}, q\right)=\Gamma\left(P, q+\frac{\partial W^{(s)}}{\partial P}\right)
$$

and if we expand $H$ and $\Gamma$ in a Taylor series about $P$ and $q$, and then collect and equate all terms of equal degree, the following set of equations, equivalent to Eq. (7), is obtained:

$$
\begin{aligned}
& H^{(i)}(P, q)=\Gamma^{(i)}(P, q), \quad i<s, \\
& D W^{(s)}(P, q)=\Gamma^{(s)}(P, q)-H^{(s)}(P, q), \quad i=s, \\
& \Gamma^{(i)}(P, q)= H^{(i)}(P, q)+\sum_{j} \frac{1}{j !} \\
& \times {\left[\left(\frac{\partial W^{(s)}}{\partial q}\right)^{j} \frac{\partial^{j} H^{(l)}}{\partial P^{j}}-\left(\frac{\partial W^{(s)}}{\partial P^{j}}\right)^{j}\left(\frac{\partial^{j} \Gamma^{(l)}}{\partial q^{j}}\right)\right], } \\
& i>s,
\end{aligned}
$$

where $j$ is the vector $\left(j_{1}, j_{2}\right)$ restricted such that

$$
\begin{aligned}
& l-|j|+|j|(s-1)=i, \\
& 1<|j|<l<i, \\
& l \geqslant 2, \quad s \geqslant 3,
\end{aligned}
$$

and $j !=j_{1} ! j_{2} !$. In these equations,

$$
\left.H^{(i)}(P, q) \equiv H^{(f)}(p, q)\right|_{p=P}
$$

and

$$
\left.\Gamma^{(i)}(P, q) \equiv \Gamma^{(i)}(P, Q)\right|_{Q=q},
$$

as arises from the Taylor expansion.

Equation (8a) shows that the transformation of degree $s$ leaves lower degree terms unaffected; Eq. (8b) is the equation to be solved for $W^{(s)}$, and when this is done, $\mathrm{Eq}$. (8c) gives the higher degree terms in the new Hamiltonian.

In order to solve Eq. (8b) for $W^{(s)}$, we temporarily make a transformation to variables in which $D$ is diagonal: Define

$$
\begin{aligned}
& P_{k}=2^{-1 / 2}\left(\eta_{k}+i \xi_{k}\right), \\
& q_{k}=i 2^{1 / 2}\left(\eta_{k}-i \xi_{k}\right) .
\end{aligned}
$$

Under this transformation $D-\tilde{D}$, where

$$
\tilde{D}(\eta, \xi)=i \sum_{k} \omega_{k}\left(\xi_{k} \frac{\partial}{\partial \xi_{k}}-\eta_{k} \frac{\partial}{\partial \eta_{k}}\right) .
$$

It follows by inspection that functions of the form

$$
\Phi_{l_{1} l_{2} m_{1} m_{2}} \equiv \eta_{1}^{l_{1}} \eta_{2}^{l_{2}} \xi_{1}^{m_{1}} \xi_{2}^{m_{2}}
$$

are eigenfunctions of $\tilde{D}$ with eigenvalues

$$
i \sum_{k} \omega_{k}\left(m_{k}-l_{k}\right)
$$

i. e.,

$$
\tilde{D} \Phi_{l_{1} l_{2} m_{1} m_{2}}=\left[i \sum_{k} \omega_{k}\left(m_{k}-l_{k}\right)\right] \Phi_{l_{1} l_{2} m_{1} m_{2}} .
$$

Consequently,

$$
\tilde{D}^{-1} \Phi_{l_{1} l_{2} m_{1} m_{2}}=\left[i \sum_{k} \omega_{k}\left(m_{k}-l_{k}\right)\right]^{-1} \Phi_{l_{1} l_{2} m_{1} m_{2}} .
$$

Under the transformation discussed here, Eq. (8b) becomes

$$
\tilde{D} \tilde{W}^{(s)}=\tilde{\Gamma}^{(s)}-\tilde{H}^{(s)} .
$$

This equation may now be solved for $\tilde{W}^{(s)}$, operating with $D^{-1}$ on both sides

$$
\tilde{W}^{(s)}=\tilde{D}^{-1}\left(\tilde{\Gamma}^{(s)}-\tilde{H}^{(s)}\right) \text {. }
$$

$\tilde{H}^{(s)}$ is a known function. However, so far, $\tilde{\Gamma}^{(s)}$ has been unspecified. $\tilde{\Gamma}^{(s)}$ is now determined from the requirement that $\bar{W}^{(s)}$ be finite. Clearly, $\tilde{\Gamma}^{(s)}$ must be chosen so as to exactly cancel any terms in $\tilde{H}^{(s)}$ which would give a vanishing denominator in $\mathrm{Eq}$. (13b). So long as the frequencies are incommensurable, the only terms that must appear in $\tilde{\Gamma}^{(s)}$ are those for which $m_{k}=l_{k}$ for all $k$; those terms are

$\left(i \eta_{1} \xi_{1}\right)^{m_{1}}\left(i \eta_{2} \xi_{2}\right)^{m_{2}}=\left[\frac{1}{2}\left(P_{1}^{2}+q_{1}^{2}\right)\right]^{m_{1}}\left[\frac{1}{2}\left(P_{2}^{2}+q_{2}^{2}\right)\right]^{m_{2}}$.

Such terms are called null space terms, and the remaining terms are called range space terms. Therefore, if $\tilde{H}^{(s)}$ is separated into null space terms $\tilde{N}^{(s)}$ and range space terms $\bar{R}^{(s)}$,

$$
\tilde{H}^{(s)}=\bar{N}^{(s)}+\tilde{R}^{(s)} \text {, }
$$

and if we require $\tilde{\Gamma}^{(s)}$ to cancel the null space terms in $\tilde{H}^{(s)}$, Eq. (15) results in

$$
\begin{aligned}
& \tilde{\Gamma}^{(s)}=\tilde{N}^{(s)}, \\
& \tilde{W}^{(s)}=\tilde{D}^{-1} \tilde{R}^{(s)} .
\end{aligned}
$$

Having obtained $\tilde{\Gamma}^{(s)}$ and $\tilde{W}^{(s)}$, we can use the inverse equations to Eq. (10) to obtain $\Gamma^{(s)}$ and $W^{(s)}$. $\Gamma^{(s)}$ will be composed only of terms such as in Eq. (16) (as mentioned, provided that the frequencies $\omega_{k}$ are incommensurable). Note that arbitrary null space terms could be added to $W$, but following Gustavson, we omit such terms. The above method for constructing the normal form simultaneously satisfies the definition of a normal form, and serves to eliminate exactly those terms in $H^{(s)}$ which would cause divergence of the equation for 
$W^{(s)}[$ Eq. (15)].

Now that degree $s$ terms in the Hamiltonian have been transformed into the normal form $\Gamma^{(s)}, W^{(s)}$ must be used in Eq. (8c) to calculate higher degree terms in the new Hamiltonian. The process can then be repeated to normalize terms of degree $s+1$. It is found that the normal form contains only terms of even degree.

As an example, we studied the Hamiltonian ${ }^{10(a),(b), 12(a),(b)}$

$$
\begin{aligned}
H & =\frac{1}{2}\left(p_{1}^{2}+p_{2}^{2}+\omega_{1}^{2} q_{1}^{2}+\omega_{2}^{2} q_{2}^{2}\right)+\lambda q_{2}\left(q_{1}^{2}+\eta q_{2}^{2}\right) \\
& =H^{(2)}+H^{(3)},
\end{aligned}
$$

where $p$ is momentum, and $q$ is a coordinate. The second degree terms $H^{(2)}$ can be brought into Birkhoff's normal form by the transformation

$$
\begin{aligned}
& p_{k}-\omega_{k}^{1 / 2} p_{k}, \\
& q_{k}-\omega_{k}^{-1 / 2} q_{k},
\end{aligned}
$$

giving

$$
\begin{aligned}
& H^{(2)}=\sum_{k=1}^{2} \frac{1}{2}\left(p_{k}^{2}+q_{k}^{2}\right) \omega_{k}, \\
& H^{(3)}=\frac{\lambda}{\omega_{1} \omega_{2}^{1 / 2}} q_{1}^{2} q_{2}+\frac{\lambda \eta}{\omega_{2}^{3 / 2}} q_{2}^{3} .
\end{aligned}
$$

Applying the above procedure, the normal form obtained through degree four with

$$
\begin{array}{ll}
\omega_{1}=1.3, & \lambda=-0.1, \\
\omega_{2}=0.7, \quad \eta=0.1
\end{array}
$$

is given by

$$
\begin{aligned}
\Gamma= & \frac{1}{2}\left[1.3\left(P_{1}^{2}+Q_{1}^{2}\right)+0.7\left(P_{2}^{2}+Q_{2}^{2}\right)\right]-1.56185 \\
& \times 10^{-3}\left[\frac{1}{2}\left(P_{2}^{2}+Q_{2}^{2}\right)\right]^{2}-1.023323 \times 10^{-2}\left[\frac{1}{4}\left(P_{2}^{2}+Q_{2}^{2}\right)\left(P_{1}^{2}+Q_{1}^{2}\right)\right] \\
& -5.80199 \times 10^{-3}\left[\frac{1}{2}\left(P_{1}^{2}+Q_{1}^{2}\right)\right]^{2} .
\end{aligned}
$$

Once the transformation to Birkhoff's normal form is made, the harmonic oscillator terms can be transformed to action variables via the canonical transformation

$$
\begin{aligned}
& P_{k}=\left(\frac{J_{k}}{\pi}\right)^{1 / 2} \cos 2 \pi w_{k}, \\
& Q_{k}=\left(\frac{J_{k}}{\pi}\right)^{1 / 2} \sin 2 \pi w_{k},
\end{aligned}
$$

which results in

$$
\frac{1}{2}\left(P_{k}^{2}+Q_{k}^{2}\right)=J_{k} / 2 \pi \text {. }
$$

For the example just given, the normal form [Eq. (23)] becomes [through terms of degree four in $(Q, P)$ ]

$$
\begin{aligned}
\Gamma= & 1.3\left(J_{1} / 2 \pi\right)+0.7\left(J_{2} / 2 \pi\right)-1.56185 \times 10^{-3}\left(J_{2} / 2 \pi\right)^{2} \\
& -1.023323 \times 10^{-2}\left(J_{1} J_{2} / 4 \pi^{2}\right)-5.80199 \times 10^{-3}\left(J_{1} / 2 \pi\right)^{2} .
\end{aligned}
$$

Once the classical motion is expressed in terms of action variables, semiclassical quantization is straightforward.

The above treatment is satisfactory provided that the frequenci es are incommensurable, but a problem arises if they are commensurable in low order. For example, assume $\omega_{1}=2 \omega_{2}$, and consider Eq. (13a) for $\Phi_{1002}=\eta_{1} \xi_{2}^{2}$ :

$$
\begin{aligned}
\bar{D} \eta_{1} \xi_{2}^{2} & =\left[\omega_{1}(-1)+\omega_{2}(2)\right] \eta_{1} \xi_{2}^{2} . \\
& =0
\end{aligned}
$$

Therefore, $\tilde{D}^{-1} \eta_{1} \xi_{2}^{2}$ would diverge.

In order to avoid this occurrence, $\tilde{\Gamma}^{(s)}$ must be chosen to cancel any such additional terms in $H^{(s)}$. A side from this change, the procedure is identical to that for the incommensurable case.

The second Hamiltonian which we treated is given by $^{10(c), 8}$

$$
H=\frac{1}{2}\left(p_{1}^{2}+p_{2}^{2}\right)+\frac{1}{2}\left(q_{1}^{2}+q_{2}^{2}\right)+\lambda q_{2}\left(q_{1}^{2}+\eta q_{2}^{2}\right),
$$

where

$$
\begin{aligned}
& \lambda=0.0125, \\
& \eta=-1 / 3 .
\end{aligned}
$$

The normal form for this Hamiltonian is (through fourth degree)

$$
\begin{aligned}
\Gamma= & \frac{1}{2}\left[\left(P_{1}^{2}+Q_{1}^{2}\right)+\left(P_{2}^{2}+Q_{2}^{2}\right)\right]-5.2083 \times 10^{-4}\left[\frac{1}{2}\left(P_{2}^{2}+Q_{2}^{2}\right)\right]^{2} \\
& +4.1667 \times 10^{-4}\left[\frac{1}{4}\left(P_{2}^{2}+Q_{2}^{2}\right)\left(P_{1}^{2}+Q_{1}^{2}\right)\right]-5.2083 \\
& \times 10^{-4}\left[\frac{1}{2}\left(P_{1}^{2}+Q_{1}^{2}\right)\right]^{2}-3.6459 \times 10^{-4}\left[\left(P_{2} P_{1}+Q_{2} Q_{1}\right)^{2}\right. \\
& \left.-\left(P_{2} Q_{1}-Q_{2} P_{1}\right)^{2}\right] .
\end{aligned}
$$

Comparison with Eq. (23) for an incommensurable case shows that cross terms have entered for the commensurable case.

We now wish to express this normal form in terms of angle-action variables. If the transformation Eqs. (24) are applied to this normal form (29), we obtain

$$
\begin{aligned}
\Gamma= & (1 / 2 \pi)\left(J_{1}+J_{2}\right)-5.2083 \times 10^{-4}\left(J_{2} / 2 \pi\right)^{2}+4.1667 \\
& \times 10^{-4}\left(J_{1} J_{2} / 4 \pi^{2}\right)-5.2083 \times 10^{-4}\left(J_{1} / 2 \pi\right)^{2} \\
& -3.6459 \times 10^{-4}\left(J_{1} J_{2} / \pi^{2}\right) \cos \left[4 \pi\left(w_{1}-w_{2}\right)\right] .
\end{aligned}
$$

It can be seen that, while the unperturbed terms depend only on the $J$ 's, the higher degree terms depend on the $w$ 's. Therefore, Eqs. (24) do not result in a transformation to angle-action variables. However, the additional canonical transformation given by

$$
\begin{array}{ll}
J_{2}=\frac{1}{2}\left(J+J^{\prime}\right), & w_{2}=\left(w+w^{\prime}\right), \\
J_{1}=\frac{1}{2}\left(J-J^{\prime}\right), & w_{1}=\left(w-w^{\prime}\right),
\end{array}
$$

will yield a form which is cyclic in $w$, so the canonically conjugate momentum $J$ is constant. This two-step process can be carried out in one step using the canonical transformation

$$
\begin{aligned}
& P_{2}=\left(\frac{J+J^{\prime}}{2 \pi}\right)^{1 / 2} \cos \left[2 \pi\left(w+w^{\prime}\right)\right], \\
& Q_{2}=\left(\frac{J+J^{\prime}}{2 \pi}\right)^{1 / 2} \sin \left[2 \pi\left(w+w^{\prime}\right)\right], \\
& P_{1}=\left(\frac{J-J^{\prime}}{2 \pi}\right)^{1 / 2} \cos \left[2 \pi\left(w-w^{\prime}\right)\right], \\
& Q_{1}=\left(\frac{J-J^{\prime}}{2 \pi}\right)^{1 / 2} \sin \left[2 \pi\left(w-w^{\prime}\right)\right] .
\end{aligned}
$$

It is found that all orders of the normal form are then cyclic in the variable $w$. The complete result, through degree 8 terms for the Hamiltonian (28), is 


$$
\begin{aligned}
\Gamma^{(2)}= & J / 2 \pi \\
\Gamma^{(4)}= & -\frac{9.1146 \times 10^{-4}}{\pi^{2}}\left(J^{2}-J^{\prime 2}\right) \cos \left(8 \pi w^{\prime}\right)-\frac{3.9063 \times 10^{-4}}{\pi^{2}} J^{2}-\frac{9.1146 \times 10^{-4}}{\pi^{2}} J^{2}, \\
\Gamma^{(6)}= & \frac{4.7 \times 10^{-7}}{\pi^{3}}\left(J^{3}-J J^{\prime 2}\right) \cos \left(8 \pi w^{\prime}\right)+\frac{1.139 \times 10^{-5}}{\pi^{3}}\left(J^{2} J^{\prime}-J^{\prime 3}\right) \cos \left(8 \pi w^{\prime}\right) \\
& -\frac{3.50 \times 10^{-6}}{\pi^{3}} J^{3}+\frac{1.139 \times 10^{-5}}{\pi^{3}} J^{2} J^{\prime}+\frac{4.7 \times 10^{-7}}{\pi^{3}} J J^{\prime 2}-\frac{1.899 \times 10^{-5}}{\pi^{3}} J^{\prime 3}, \\
\Gamma^{(8)}= & \frac{5.6 \times 10^{-8}}{\pi^{4}}\left(J^{2}-J^{\prime 2}\right)^{2} \cos \left(16 \pi w^{\prime}\right)-\frac{4.6 \times 10^{-8}}{\pi^{4}} J^{4} \cos \left(8 \pi w^{\prime}\right)+\frac{2.72 \times 10^{-7}}{\pi^{4}} J^{2} J^{\prime 2} \cos \left(8 \pi w^{\prime}\right) \\
& -\frac{2.26 \times 10^{-7}}{\pi^{4}} J^{\prime 4} \cos \left(8 \pi w^{\prime}\right)+\frac{1.77 \times 10^{-7}}{\pi^{4}}\left(J^{3} J^{\prime}-J J^{\prime 3}\right) \cos \left(8 \pi w^{\prime}\right)-\frac{4.4 \times 10^{-8}}{\pi^{4}} J^{4}+\frac{1.77 \times 10^{-7}}{\pi^{4}} J^{3} J^{\prime} \\
& -\frac{1.59 \times 10^{-7}}{\pi^{4}} J^{2} J^{\prime 2}-\frac{2.96 \times 10^{-7}}{\pi^{4}} J J^{\prime 3}+\frac{1.69 \times 10^{-7}}{\pi^{4}} J^{\prime 4},
\end{aligned}
$$

where $\Gamma^{(2)}$ corresponds to terms of degree 2 in $(P, Q)$, etc.

$J$ can be shown to be an action variable by noting that

$$
\oint \sum_{k} P_{k} d Q_{k}=J
$$

where the phase integral is evaluated over a path in $(Q, P)$ space generated by letting $w$ increase by unity (bringing $Q, P$ through one cycle) while holding $\left(J^{\prime}, w^{\prime}\right)$ fixed. This follows by observing that

$$
\begin{aligned}
& d Q_{2}=\left(\frac{J+J^{\prime}}{2 \pi}\right)^{1 / 2} \cos \left[2 \pi\left(w+w^{\prime}\right)\right](2 \pi d w), \\
& d Q_{1}=\left(\frac{J-J^{\prime}}{2 \pi}\right)^{1 / 2} \cos \left[2 \pi\left(w-w^{\prime}\right)\right](2 \pi d w),
\end{aligned}
$$

and by replacing $P_{1}, P_{2}$ by expressions given in Eqs. (32a) and (32c).

However, $J^{\prime}$ is not an action variable, since $w^{\prime}$ appears in the Hamiltonian. We therefore see that, for the commensurable case, the normal form is not an analytic expression in terms of angle-action variables. Nevertheless, by transforming one pair of the variables into angle-action form, we have effectively reduced $\Gamma$ to a single degree of freedom, since the action $J$ can be regarded as a fixed parameter. This will allow us to quantize $J$ directly, and satisfy the other quantization condition by numerically quantizing a one-dimensional phase integral. After the normal form is obtained, the numerical effort involved is only slightly greater than that involved in one-dimensional WKB calculations.

There is one additional note in regard to generating the normal form. Although the method is straightforward in principle, even for a relatively simple Hamiltonian, the algebraic manipulations involved can rapidly get out of hand. (For example, expanded through eighth degree, the many higher order terms of the Hamiltonian generated while constructing the normal form lead to intermediate forms for the Hamiltonian containing almost 500 terms.) We found it most convenient to carry out the sequence of canonical transformations using MACSYMA, ${ }^{19}$ a computer language that permits analytical calculations. The calculation of the normal form for the incommensurable case required less than $4 \mathrm{~min}$ CPU time on a PDP 10 when done using single precision, and 24 min CPU time when done using extended precision. Calculations for the commensurable case took slightly more CPU time.

\section{QUANTIZING THE NORMAL FORM}

In this section, we calculate a semiclassical approximation to an energy spectrum by quantizing the normal form, and we compare the results to essentially exact quantum mechanical results that were obtained for us by D. Noid. ${ }^{20}$

Quantizing the incommensurable case is straightfor ward. Since the normal form can be expressed entirely in terms of action variables, we merely have to replace those action variables by an appropriate multiple of $h$ (half- or full-integral quantization), and the result is a power series in the quantum numbers. The commensurable case is less straightforward. Since the normal form is expressed in terms of one action variable, we can again quantize that action variable by replacing it by the appropriate multiple of $h$, and the second quantum condition is provided by numerically evaluating and quantizing a phase integral.

Again, it should be borne in mind that we are ignoring the full complexity of classical motion (i.e., the irregular trajectories embedded among the multiply periodic ones). Equivalently, we are ignoring the fact that the normal form does not converge. Nevertheless, by truncating the normal form, we are able to obtain remarkable agreement with quantum mechanics, as shown below.

\section{A. The incommensurable case}

After obtaining the normal form, and expressing it in terms of action variables, we merely have to make the following replacement:

$$
\frac{1}{2}\left(P_{k}^{2}+Q_{k}^{2}\right)-\frac{J_{k}}{2 \pi}-\left(n_{k}+\frac{1}{2}\right) h
$$

where the quantum number $n_{k}$ is an integer. Notice that half-integral quantization has been chosen here. Refer- 
ence to Eqs. (24a) and (24b) shows that $J_{k}$ is given by

$$
J_{k}=\oint P_{k} d Q_{k} \quad \text { (no sum), }
$$

where the phase integral is evaluated over a path generated by allowing the angle variable $w_{k}$ to increase by unity (bringing $Q_{k}, P_{k}$ through one cycle) while holding $w_{k^{\prime}}\left(k^{\prime} \neq k\right)$ constant. In $(Q, P)$ space, the resultant path will have two turning points (zeros of $P_{k}$ ), and $Q_{k^{\prime}}$ and $P_{k^{\prime}}\left(k^{\prime} \neq k\right)$ may vary, but must return to their orig inal values without passing through a full cycle (otherwise $w_{k^{\prime}}$ would have increased by unity). Half-integral quantization therefore follows. Also, in the unperturbed limit, the normal form simply describes two uncoupled harmonic oscillators, for which half-integral quantization is correct. The quantization for the perturbed case therefore reduces unchanged to the correct quantization in the unperturbed limit.

For the Hamiltonian (20), with parameters

$$
\begin{array}{ll}
\omega_{1}=1.3, & \lambda=-0.1, \\
\omega_{2}=0.7, & \eta=0.1,
\end{array}
$$

the quantized normal form is (up to harmonic oscillator terms of degree 8)

$$
\begin{aligned}
\Gamma\left[\left(n_{1}+\frac{1}{2}\right) h,\left(n_{2}+\frac{1}{2}\right) h\right]= & 1.3\left(n_{1}+\frac{1}{2}\right)+0.7\left(n_{2}+\frac{1}{2}\right)-0.00156185\left(n_{2}+\frac{1}{2}\right)^{2}-0.01023323\left(n_{1}+\frac{1}{2}\right)\left(n_{2}+\frac{1}{2}\right)-0.00580199\left(n_{1}+\frac{1}{2}\right)^{2} \\
& -0.00001092\left(n_{2}+\frac{1}{2}\right)^{3}-0.00017719\left(n_{1}+\frac{1}{2}\right)\left(n_{2}+\frac{1}{2}\right)^{2}-0.00027327\left(n_{1}+\frac{1}{2}\right)^{2}\left(n_{2}+\frac{1}{2}\right)-0.00008625\left(n_{1}+\frac{1}{2}\right)^{3} \\
& -0.00000013\left(n_{2}+\frac{1}{2}\right)^{4}-0.00000529\left(n_{1}+\frac{1}{2}\right)\left(n_{2}+\frac{1}{2}\right)^{3}-0.00001183\left(n_{1}+\frac{1}{2}\right)^{2}\left(n_{2}+\frac{1}{2}\right)^{2} \\
& -0.00001071\left(n_{1}+\frac{1}{2}\right)^{3}\left(n_{2}+\frac{1}{2}\right)-0.00000229\left(n_{1}+\frac{1}{2}\right)^{4}
\end{aligned}
$$

where $\hbar=1$ here.

Table I compares the energy levels predicted by this formula with quantum mechanical values calculated with a $30 \times 30$ (900 function) harmonic oscillator basis set. Two percentage errors are listed. The first $\left(\Delta E_{\mathrm{rel}}\right)$ is the percent error of the correction to the unperturbed energy [i.e., $\Delta E_{\text {rol }}=\left(E_{B N F}-E_{Q N}\right) /\left(E_{0}-E_{Q M}\right)$ ]. This error parameter is a useful measure of the improvement that a semiclassical calculation gives over a zeroth order approximation which treats the system as a set of uncoupled harmonic oscillators. The second $\left(\Delta E_{\mathrm{abs}}\right)$ is the percent error of the energy value $\left[\Delta E_{\mathrm{abs}}=\left(E_{B N F}\right.\right.$ $\left.\left.-E_{Q \mu}\right) / E_{Q \mu}\right]$. As shown, $\Delta E_{\text {rol }}$ is less than $2 \%$ through level 42 , and less than about $7 \%$ over the entire spectrum. $\Delta E_{\text {abs }}$ is less than about $0.08 \%$ through level 42 , and less than about $0.5 \%$ over the entire spectrum.

It will be seen that the absolute error of the semiclassical value is comparable to the spacing between levels for states near the escape energy $\left(E_{\text {esc }}=11.5\right)$. However, even ignoring the values based on the normal form, it is easy to verify that all of the quantum values fall into a regular pattern that is consistent with the given assignments. (There are some sets of nearly degenerate states that are difficult to identify definitively, but the given assignments appear to be most compatible with the pattern of other levels.)

Caution is required in drawing general conclusions about irregular spectra from this example, because the region of irregular classical behavior has not been extensively studied.

\section{B. The commensurable case}

To quantize the normal form given in Eq. (33), let us begin by quantizing $J$ :

$$
J=\oint_{c} \sum_{k} P_{k} d Q_{k}
$$

where the path $c$ is defined by letting the angle variable $w$ increase by unity, holding $w^{\prime}$ fixed. In this case, there appears to be no obvious argument telling how many caustics such an integration path will touch. We therefore found it necessary to obtain the correct quantization by numerically generating an integration path in $(Q, P)$ space using Eqs. (32) with $w$ varying. (This illustrates a problem with methods which do not display integration paths; the proper quantization sometimes can be determined only by actually counting the number of caustics touched by the integration path.) As was found by Noid and Marcus, two classes of motion appear-rotations and librations. Figure 1 shows the caustics for each type of trajectory, and the paths labeled $A$ are the ones obtained by varying $w$. For both classes, the integration path touches four caustics, and Keller's quantization prescription ${ }^{21}$ therefore requires full integral quantization

$$
J=(n+1) h \text {. }
$$

It will be noted that, in the unperturbed limit, the normal form again represents two uncoupled harmonic oscillators (with equal frequencies in this case). The correct quantization is half-integral for each oscillator; however, because the frequencies are equal, the actions for each oscillator can be directly added, yielding Eq. (39). We therefore find that the quantization for the perturbed case again reduces unchanged to the correct quantization in the unperturbed limit.

For the second quantum condition, we quantize the phase integral

$$
\oint_{c^{\prime}} J^{\prime} d w^{\prime}=\oint_{c^{\prime}} \sum_{k} P_{k} d Q_{k},
$$

where the path $c^{\prime}$ is defined by allowing $w^{\prime}$ to vary over one cycle, holding $w$ fixed. Again, we must determine how many caustics the integration path touches, and we use Eqs. (32) to generate an integration path in $(Q, P)$ space as $w^{\prime}$ varies. This time the process is complicated by the fact that $J^{\prime}$ varies with $w^{\prime}$. In order to find the dependence of $J^{\prime}$ on $w^{\prime}$, we use the normal form (33). 
TABLE I. Energy levels for an incommensurable case.

\begin{tabular}{|c|c|c|c|c|c|c|c|}
\hline$n$ & $N_{0}$ & $N_{1}$ & $E_{\text {BGNF }}{ }^{2}$ & $E_{Q N}^{b}$ & $E_{0}{ }^{c}$ & $\Delta E_{r e l}^{d}$ & $\Delta E_{\mathrm{abs}}{ }^{\bullet}$ \\
\hline 1 & 0 & 0 & 0.9955 & 0.9955 & 1.0000 & $0 \%$ & $0 \%$ \\
\hline 2 & 0 & 1 & 1.6870 & 1.6870 & 1. 7 & $0 \%$ & $0 \%$ \\
\hline 3 & 1 & 0 & 2.2782 & 2.2781 & $2: 3$ & $0.5 \%$ & $0.004 \%$ \\
\hline 4 & 0 & 2 & 2. 3750 & 2.3750 & 2.4 & $0 \%$ & $0 \%$ \\
\hline 5 & 1 & 1 & 2.9584 & 2.9584 & 3.0 & $0 \%$ & $0 \%$ \\
\hline 6 & 0 & 3 & 3.0595 & 3.0596 & 3.1 & $-0.2 \%$ & $-0.003 \%$ \\
\hline 7 & 2 & 0 & 3.5480 & 3.5479 & 3.6 & $0.2 \%$ & $0.003 \%$ \\
\hline 8 & 1 & 2 & 3.6348 & 3.6347 & 3.7 & $0.2 \%$ & $0.003 \%$ \\
\hline 9 & 0 & 4 & 3. 7404 & 3. 7404 & 3.8 & $0 \%$ & $0 \%$ \\
\hline 10 & 2 & 1 & 4.2164 & 4.2162 & 4.3 & $0.2 \%$ & $0.005 \%$ \\
\hline 11 & 1 & 3 & 4.3071 & 4.3069 & 4.4 & $0.2 \%$ & $0.005 \%$ \\
\hline 12 & 0 & 5 & 4.4176 & 4.4176 & 4.5 & $0 \%$ & $0 \%$ \\
\hline 13 & 3 & 0 & 4.8045 & 4. 8043 & 4. 9 & $0.2 \%$ & $0.004 \%$ \\
\hline 14 & 2 & 2 & 4. 8803 & 4. 8799 & 5.0 & $0.3 \%$ & $0.008 \%$ \\
\hline 15 & 1 & 4 & 4.9753 & 4.9749 & 5.1 & $0.3 \%$ & $0.008 \%$ \\
\hline 16 & 0 & 6 & 5.0910 & 5.0909 & 5.2 & $0.1 \%$ & $0.002 \%$ \\
\hline 17 & 3 & 1 & 5.4601 & 5.4597 & 5.6 & $0.3 \%$ & $0.007 \%$ \\
\hline 18 & 2 & 3 & 5.5397 & 5.5390 & 5.7 & $0.4 \%$ & $0.013 \%$ \\
\hline 19 & 1 & 5 & 5.6393 & 5.6385 & 5.8 & $0.5 \%$ & $0.014 \%$ \\
\hline 20 & 0 & 7 & 5.7605 & 5.7601 & 5.9 & $0.3 \%$ & $0.007 \%$ \\
\hline 21 & 4 & 0 & 6.0468 & 6.0463 & 6.2 & $0.3 \%$ & $0.008 \%$ \\
\hline 22 & 3 & 2 & 6.1108 & 6.1099 & 6.3 & $0.5 \%$ & $0.015 \%$ \\
\hline 23 & 2 & 4 & 6.1944 & 6.1931 & 6.4 & $0.6 \%$ & $0.021 \%$ \\
\hline 24 & 1 & 6 & 6.2989 & 6.2975 & 6.5 & $0.7 \%$ & $0.022 \%$ \\
\hline 25 & 0 & 8 & 6.4260 & 6.4253 & 6.6 & $0.5 \%$ & $0.012 \%$ \\
\hline 26 & 4 & 1 & 6.6889 & 6.6878 & 6.9 & $0.5 \%$ & $0.016 \%$ \\
\hline 27 & 3 & 3 & 6.7564 & 6.7546 & 7.0 & $0.7 \%$ & $0.027 \%$ \\
\hline 28 & 2 & 5 & 6.8443 & 6.8419 & 7.1 & $0.9 \%$ & $0.035 \%$ \\
\hline 29 & 1 & 7 & 6.9540 & 6.9515 & 7.2 & $1.0 \%$ & $0.036 \%$ \\
\hline 30 & 0 & 9 & 7.0873 & 7.0861 & 7.3 & $0.6 \%$ & $0.017 \%$ \\
\hline 31 & 5 & 0 & 7.2742 & 7.2730 & 7.5 & $0.5 \%$ & $0.017 \%$ \\
\hline 32 & 4 & 2 & 7.3255 & 7.3234 & 7.6 & $0.7 \%$ & $0.029 \%$ \\
\hline 33 & 3 & 4 & 7.3967 & 7.3935 & 7.7 & $1.0 \%$ & $0.043 \%$ \\
\hline 34 & 2 & 6 & 7.4891 & 7.4850 & 7.8 & $1.3 \%$ & $0.055 \%$ \\
\hline 35 & 1 & 8 & 7.6044 & 7.6003 & 7.9 & $1.6 \%$ & $0.054 \%$ \\
\hline 36 & 0 & 10 & 7.7445 & 7.7423 & 8.0 & $0.9 \%$ & $0.028 \%$ \\
\hline 37 & 5 & 1 & 7.9019 & 7.8996 & 8.2 & $0.8 \%$ & $0.029 \%$ \\
\hline 38 & 4 & 3 & 7.9564 & 7.9524 & 8.3 & $1.2 \%$ & $0.050 \%$ \\
\hline 39 & 3 & 5 & 8.0315 & 8.0259 & 8.4 & $1.5 \%$ & $0.070 \%$ \\
\hline 40 & 2 & 7 & 8.1288 & 8.1220 & 8.5 & $1.8 \%$ & $0.084 \%$ \\
\hline 41 & 1 & 9 & 8. 2500 & 8.2435 & 8.6 & $1.8 \%$ & $0.079 \%$ \\
\hline 42 & 0 & 11 & 8. 3973 & 8.3939 & 8.7 & $1.1 \%$ & $0.041 \%$ \\
\hline 43 & 6 & 0 & 8. 4858 & 8.4835 & 8.8 & $0.7 \%$ & $0.062 \%$ \\
\hline 44 & 5 & 2 & 8.5235 & 8.5190 & 8.9 & $1.2 \%$ & $0.053 \%$ \\
\hline 45 & 4 & 4 & 8.5814 & 8.5743 & 9.0 & 1. $7 \%$ & $0.083 \%$ \\
\hline 46 & 3 & 6 & 8.6607 & 8.6513 & 9.1 & $2.1 \%$ & $0.109 \%$ \\
\hline 47 & 2 & 8 & 8.7632 & 8.7524 & 9.2 & $2.4 \%$ & $0.123 \%$ \\
\hline 48 & 1 & 10 & 8.8908 & 8.8805 & 9.3 & $2.5 \%$ & $0.116 \%$ \\
\hline 49 & 0 & 12 & 9.0456 & 9.0403 & 9.4 & $1.5 \%$ & $0.059 \%$ \\
\hline 50 & 6 & 1 & 9.0982 & 9.0934 & 9.5 & $1.2 \%$ & $0.053 \%$ \\
\hline 51 & 5 & 3 & 9.1387 & 9.1305 & 9.6 & $1.7 \%$ & $0.090 \%$ \\
\hline 52 & 4 & 5 & 9.2001 & 9. 1882 & 9.7 & $2.3 \%$ & $0.130 \%$ \\
\hline 53 & 3 & 7 & 9.2840 & 9.2689 & 9.8 & $2.8 \%$ & $0.163 \%$ \\
\hline 54 & 2 & 9 & 9.3921 & 9.3752 & 9.9 & $3.2 \%$ & $0.180 \%$ \\
\hline 55 & 1 & 11 & 9.5264 & 9.5108 & 10.0 & $3.2 \%$ & $0.164 \%$ \\
\hline 57 & 0 & 13 & 9.6894 & 9.6812 & 10.1 & $2.0 \%$ & $0.085 \%$ \\
\hline 56 & 7 & 0 & 9.6808 & 9.6762 & 10.1 & $1.0 \%$ & $0.048 \%$ \\
\hline 58 & 6 & 2 & 9.7038 & 9.6948 & 10.2 & $1.8 \%$ & $0.093 \%$ \\
\hline 59 & 5 & 4 & 9.7472 & 9.7331 & 10.3 & $2.5 \%$ & $0.145 \%$ \\
\hline 60 & 4 & 6 & 9.8125 & 9.7932 & 10.4 & $3.2 \%$ & $0.197 \%$ \\
\hline 61 & 3 & 8 & 9.9012 & 9.8776 & 10.5 & $3.8 \%$ & $0.24 \%$ \\
\hline 62 & 2 & 10 & 10.0152 & 9.9895 & 10.6 & 4. $2 \%$ & $0.26 \%$ \\
\hline 63 & 1 & 12 & 10.1568 & 10.1332 & 10.7 & 4. $1 \%$ & $0.23 \%$ \\
\hline 66 & 0 & 14 & 10.3285 & 10.3161 & 10.8 & $2.6 \%$ & $0.120 \%$ \\
\hline 64 & 7 & 1 & 10.2769 & 10.2676 & 10.8 & $1.7 \%$ & $0.091 \%$ \\
\hline 65 & 6 & 3 & 10.3023 & 10.2861 & 10.9 & $2.6 \%$ & $0.157 \%$ \\
\hline 67 & 5 & 5 & 10.3489 & 10.3253 & 11.0 & $3.5 \%$ & $0.23 \%$ \\
\hline
\end{tabular}


TABLE I (Continued)

\begin{tabular}{|c|c|c|c|c|c|c|c|}
\hline$n$ & $N_{0}$ & $N_{1}$ & $E_{B G N F^{a}}$ & $E_{Q M}{ }^{b}$ & $E_{0}{ }^{c}$ & $\Delta E_{\mathrm{rel}} \mathrm{d}$ & $\Delta E_{\mathrm{abs}}{ }^{\circ}$ \\
\hline 68 & 4 & 7 & 10.4183 & 10.3877 & 11.1 & $4.3 \%$ & $0.29 \%$ \\
\hline 69 & 3 & 9 & 10.5121 & 10.4758 & 11.2 & $5.0 \%$ & $0.35 \%$ \\
\hline 70 & 2 & 11 & 10.6326 & 10.5937 & 11.3 & $5.5 \%$ & $0.37 \%$ \\
\hline 71 & 1 & 13 & 10.7819 & 10.7463 & 11.4 & $5.4 \%$ & $0.33 \%$ \\
\hline 76 & 0 & 15 & 10.9627 & 10.9439 & 11.5 & $3.4 \%$ & $0.172 \%$ \\
\hline 72 & 8 & 0 & 10.8582 & 10.8478 & 11.4 & $1.9 \%$ & $0.096 \%$ \\
\hline 73 & 7 & 2 & 10.8654 & 10.8493 & 11.5 & $2.5 \%$ & $0.148 \%$ \\
\hline 74 & 6 & 4 & 10.8933 & 10.8657 & 11.6 & $3.8 \%$ & $0.25 \%$ \\
\hline 75 & 5 & 6 & 10.9434 & 10.9053 & 11.7 & $4.8 \%$ & $0.35 \%$ \\
\hline 77 & 4 & 8 & 11.0172 & 10.9700 & 11.8 & $5.7 \%$ & $0.43 \%$ \\
\hline 78 & 3 & 10 & 11.1166 & 11.0612 & 11.9 & $6.6 \%$ & $0.50 \%$ \\
\hline 79 & 2 & 12 & 11. 2439 & 11. 1856 & 12.0 & $7.2 \%$ & $0.52 \%$ \\
\hline 80 & 1 & 14 & 11.4014 & 11.3484 & 12.1 & $7.1 \%$ & $0.47 \%$ \\
\hline 85 & 0 & 16 & $11.5902^{t}$ & $11.5324^{p}$ & 12.2 & & \\
\hline 81 & 8 & 1 & 11. 4369 & 11.4129 & 12.1 & $3.5 \%$ & $0.21 \%$ \\
\hline 82 & 7 & 3 & 11.4460 & 11.4158 & 12.2 & $3.9 \%$ & $0.26 \%$ \\
\hline 83 & 6 & 5 & 11.4767 & 11.4325 & 12.3 & $5.1 \%$ & $0.39 \%$ \\
\hline 84 & 5 & 7 & $11.5305^{f}$ & 11.4703 & 12.4 & & \\
\hline
\end{tabular}

${ }^{2}$ Calculated using the present method.

Calculated by Don Noid. ${ }^{20}$

${ }^{d} \Delta E_{\mathrm{rel}}=\left(E_{B G N F}-E_{Q M}\right) /\left(E_{0}-E_{Q M}\right)$.

${ }^{e} \Delta E_{\mathrm{abe}}=\left(E_{B G N F}-E_{Q M}\right) / E_{Q H}$.

${ }^{c}$ Energy of uncoupled system.

${ }^{1}$ Exceeds escape energy $E_{\text {exc }}=11.5 \mathrm{eV}$.

If the value of $\Gamma$ (energy) and $J$ are chosen, Eq. (33) can be solved for $J^{\prime}$ as a function of $w^{\prime}$.

Care must be taken at this point. Notice that if $\Gamma$ were approximated by including terms only through $\Gamma^{(6)}$, then a cubic equation for $J^{\prime}$ would result; if terms through $\Gamma^{(8)}$ were included, then a quartic equation would result; and so on. We expect that as more terms of $\Gamma$ are included, $J^{\prime}$ will be expressed more accurately as a function of $w^{\prime}$. However, also an additional root is introduced for each additional degree included in $\Gamma$. In order to generate the integration path in $(Q, P)$ space, we must be able to select the correct root. We are looking for the roots whose value we know approximately when only lower degree terms are included in $\Gamma$, and whose accuracy increases as we include higher degree terms in $\Gamma$.

It was found by inspection that, if terms through $\Gamma^{(6)}$ are included, the roots to the resulting cubic equation for $J^{\prime}$ as a function of $w^{\prime}$ have a structure such as that shown in Figs. 2 and 3 (with $w=0$ ). It can be seen that for some values of $w^{\prime}$, there is only one real root $J^{\prime}$, while for other $w^{\prime}$, there are three real roots $J^{\prime}$. (The root which is shown as a wavy line in the diagram may appear above or below the closed loop.)

It was further found by investigation that if terms through $\Gamma^{(8)}$ are included, then the roots lying on the closed loops of Figs. 2 and 3 only change slightly. The other root may change considerably, and a fourth root is also introduced since the equation for $J^{\prime}$ is now quartic.

Finally, when trajectories in $(P, Q)$ space were generated, and points on those trajectories were transformed into $\left(J^{\prime}, w^{\prime}\right)$ variables, they were always found to lie on the loops shown in Figs. 2 and 3. Therefore, it was concluded that they were the physically meaningful roots. The fact that the position of the closed loop changed only slightly when $\Gamma^{(8)}$ terms were included
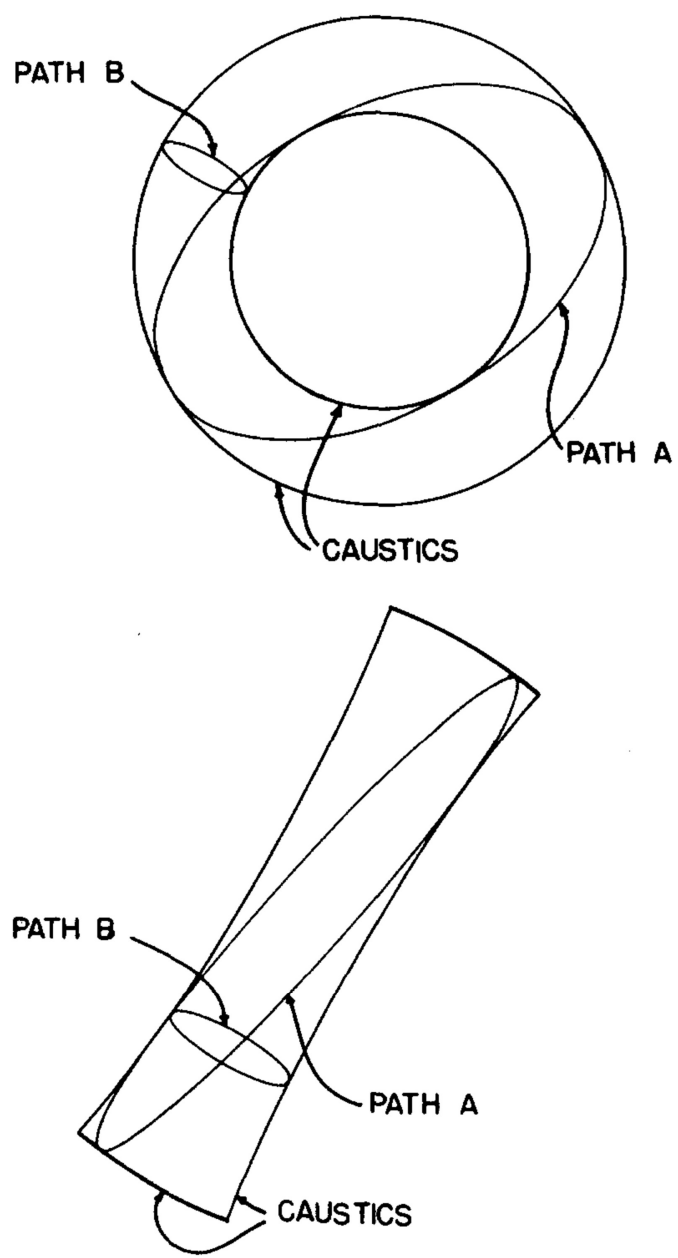

FKG. 1. (a) Schematic for quantization of rotation-type trajectories. The caustics are the boundaries between the classically allowed and classically forbidden regions: the trajectory lies within the annulus. (A) and (B) are paths for integration to obtain action variables. (b) Same as Fig. 1(a) but for libration-type trajectories. The trajectory lies within the distorted rectangle. 


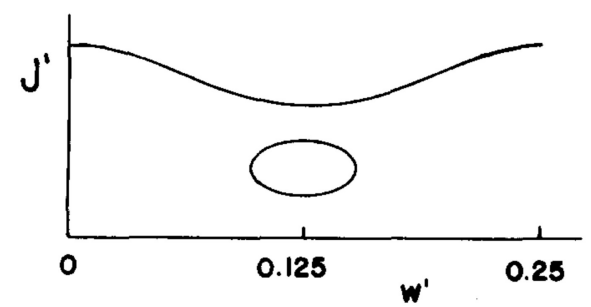

FIG. 2. $J^{\prime}$ vs $w^{\prime}$ for a rotation-type trajectory. This is obtained by regarding $J$ and $\Gamma$ as fixed parameters and numerically solving Eq. (33) for $J^{\prime}$ as a function of $w^{\prime}$.

could be taken as an indication that the truncated series for $\Gamma$ gives physically meaningful results.

One final point was observed: For the cases tested, rotations always transformed onto a loop centered on $w^{\prime}=1 / 8$ (Fig. 2), while librations always transformed onto a loop centered on $w^{\prime}=0$ (Fig. 3). Therefore, integration paths in $(Q, P)$ space for rotations were generated by varying $w^{\prime}$ over a loop centered on $w^{\prime}=1 / 8$, while paths for librations were generated by varying $w^{\prime}$ over a loop centered on $w^{\prime}=0$.

Having determined which root $J^{\prime}$ was the correct one to choose, we proceeded to see how many caustics were touched by the integration path for the phase integral (40), i. e., $w^{\prime}$. was varied over a cycle, the corresponding $J^{\prime}$ was calculated by choosing the correct root of $\Gamma=E$, and the transformation equations (32) were used to generate an integration path. This path is shown [labeled as path (B)] lying on a trajectory in Fig. 1.

Using the notation of Noid and Marcus, we see by referring to Fig. 1 that integrating around a loop centered on $w^{\prime}=1 / 8$ for a rotation case is topologically equivalent to calculating an $r$-cycle phase integral:

$$
\oint_{\text {rotation }} J^{\prime} d w^{\prime}=\oint p_{r} d r=\left(n_{r}+\frac{1}{2}\right) h .
$$

Integrating around a loop centered on $w^{\prime}=0$ for a libration case is topologically equivalent to calculating a $\theta$ cycle phase integral

$$
\oint_{1 \text { ibration }} J^{\prime} d w^{\prime}=\oint_{p_{y}} d y-\frac{1}{3} 2 \pi l h
$$

(where we have used the same quantization for the $\theta$ cycle phase integral as did Noid and Marcus, despite the fact that a proper treatment requires that tunneling be included). Notice that, in effect, the integration quantizes the area of the loops shown in Figs. 2 and 3.

The energy levels were generated using a program written in APL. The steps involved in calculating one energy level are as follows:

(1) set $J$ by selecting a value for the quantum number $n$ in Eq. (39);

(2) guess a value for the energy $E$ (the energy of the unperturbed level is a good first guess);

(3) calculate the phase integral shown in Eq. (40) numerically using values for $J^{\prime}$ provided by inverting the equation for $\Gamma$;
(4) iterate steps (2) through (4) until the value of the phase integral has converged with sufficient precision to the desired value. The successive guesses for energy are made using a linear extrapolator. Two passes must be made initially in order to extrapolate. The process converged fairly rapidly for the levels we calculated; a total of five to six passes were required for convergence.

Table II lists the levels calculated using this method for the commensurable case with Hamiltonian (28) [normal form (33)]. Comparison is made with semiclassi$\mathrm{cal}^{10}$ and quantum ${ }^{20}$ levels of Noid and Marcus. The percentage error $\Delta E_{\text {rel }}$ in the correction to the unperturbed energy is in general somewhat higher than it was for the previous incommensurable case, and for certain levels, it is very large. However, as indicated by the percentage error $\Delta E_{\text {abs }}$, the absolute error of these levels is no worse on the average than that of other levels listed; hence, the seemingly large values of $\Delta E_{\text {rel }}$ do not in fact represent serious errors. Comparison with the results for the previous incommensurable case shows that $\Delta E_{\text {abs }}$ is roughly the same in both cases for the higher energy levels, but that $\Delta E_{\text {abs }}$ is much lower in the incommensurable case for the lower energy levels. ${ }^{22}$

We also see in Table II that the present semiclassical method has succeeded in accurately predicting many energy levels well beyond the energy of transition to predominantly irregular behavior $\left(E_{\text {trans }} \simeq 9\right)$. These results are relevant to Percival's speculations on the existence of an irregular spectrum. First, we see that, of the 29 levels ${ }^{23}$ with energies between the transition energy $(\sim 9.0)$ at which irregular trajectories appear and escape energy (13.33), 17 are calculated with good accuracy by the fourth degree normal form.

For certain states having low values of $l$ (for example $l= \pm 1, n=0, n_{r}=4$ ), the present method gives no quantum level, because no conditions were found for which the phase integral (40) satisfied the quantum condition (though in some such cases the quantum condition could be approximately satisfied). Two possible explanations are (i) that this results from errors inherent in the truncation of the normal form or (ii) that this results from the use of improper quantization conditions (the conditions used do not properly account for tunneling, which may be important for librations). However, it should not be suggested that these levels belong to the irregular spectrum, because they fall into the pattern established by the regular spectrum.

There remain ten states $\left(n_{Q M}=90-99\right)$ which the eighth degree normal form is not sufficiently accurate to iden-

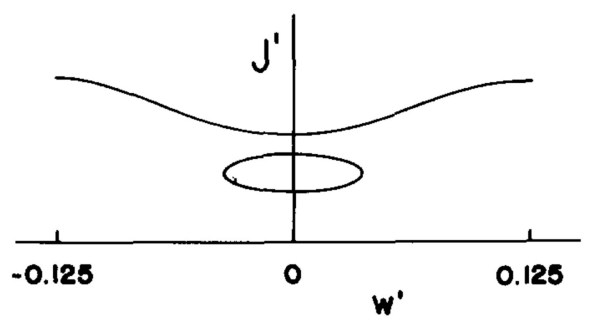

FIG. 3. Same as Fig. 2 for a libration-type trajectory. 
TABLE II. Energy levels for a commensurable case.

\begin{tabular}{|c|c|c|c|c|c|c|c|c|c|}
\hline$n_{Q M}$ & $l$ & $n$ & $n_{r}$ & $E_{0}{ }^{a}$ & $E_{Q M}{ }^{b}$ & $E_{N M}{ }^{c}$ & $E_{B G N F}{ }^{d}$ & $\Delta E_{\mathrm{r} \theta \mathrm{l}}^{\mathrm{e}}$ & $\Delta E_{\mathrm{abe}}{ }^{1}$ \\
\hline 1 & 0 & 0 & 0 & 1.0 & 0.9986 & 0.9947 & 0.9947 & $-279 \%$ & $-0.4 \%$ \\
\hline 2,3 & \pm 1 & 1 & 0 & 2.0 & 1.9901 & 1.9863 & 1.9862 & $-39 \%$ & $-0.2 \%$ \\
\hline 4 & 0 & 2 & 1 & 3.0 & 2.9562 & 2.9506 & 2.9506 & $-13 \%$ & $-0.2 \%$ \\
\hline 5,6 & \pm 2 & 2 & 0 & 3.0 & 2.9853 & 2. 9815 & 2. 9814 & $-27 \%$ & $0.13 \%$ \\
\hline 7,8 & \pm 1 & 3 & 1 & 4.0 & 3.9260 & 3.9233 & 3.9226 & $-5 \%$ & $-0.09 \%$ \\
\hline $\begin{array}{r}9 \\
10\end{array}$ & \pm 3 & 3 & 0 & 4.0 & $\begin{array}{l}3.9824 \\
3.9858\end{array}$ & 3.9803 & 3.9801 & $-25 \%$ & $-0.10 \%$ \\
\hline 11 & 0 & 4 & 2 & 5.0 & 4.8702 & 4.8573 & 4. 8577 & $-10 \%$ & $-0.3 \%$ \\
\hline 12,13 & \pm 2 & 4 & 1 & 5.0 & 4.8987 & 4.9854 & 4. 8954 & $-3 \%$ & $-0.07 \%$ \\
\hline 14,15 & \pm 4 & 4 & 0 & 5.0 & 4.9863 & 4.9821 & 4.9821 & $-31 \%$ & $-0.08 \%$ \\
\hline 16,17 & \pm 1 & 5 & 2 & 6.0 & 5.8170 & 5.816 & & & \\
\hline $\begin{array}{l}18 \\
19\end{array}$ & \pm 3 & 5 & 1 & 6.0 & $\begin{array}{l}5.8670 \\
5.8815\end{array}$ & 5.8713 & 5.8711 & $-2 \%$ & $-0.05 \%$ \\
\hline 20,21 & \pm 5 & 5 & 0 & 6.0 & 5.9913 & 5.9869 & 5.9871 & $-48 \%$ & $-0.07 \%$ \\
\hline 22 & 0 & 6 & 3 & 7.0 & 6.7379 & 6.7078 & 6.7098 & $-11 \%$ & $-0.4 \%$ \\
\hline 23,24 & \pm 2 & 6 & 2 & 7.0 & 6.7649 & 6.7709 & 6.7668 & $1 \%$ & $+0.03 \%$ \\
\hline 25,26 & \pm 4 & 6 & 1 & 7.0 & 6.8354 & 6.8500 & 6.8492 & $8 \%$ & $+0.2 \%$ \\
\hline $\begin{array}{l}27 \\
28\end{array}$ & \pm 6 & 6 & 0 & 7.0 & $\begin{array}{l}6.9989 \\
6.9994\end{array}$ & 6.9958 & 6.9951 & $-482 \%$ & $-0.05 \%$ \\
\hline 29,30 & \pm 1 & 7 & 3 & 8.0 & 7.6595 & 7.655 & & & \\
\hline $\begin{array}{l}31 \\
32\end{array}$ & \pm 3 & 7 & 2 & 8.0 & $\begin{array}{l}7.6977 \\
7.7369\end{array}$ & 7.7178 & 7.7184 & $0.4 \%$ & $0.01 \%$ \\
\hline 33,34 & \pm 5 & 7 & 1 & 8.0 & 7.8327 & 7.8289 & 7.8293 & $-2 \%$ & $-0.04 \%$ \\
\hline 35,36 & \pm 7 & 7 & 0 & 8.0 & 8.0094 & 8. 0054 & 8.0058 & $38 \%$ & $-0.04 \%$ \\
\hline 37 & 0 & 8 & 4 & 9.0 & 8.5541 & 8.4919 & 8.5003 & $-12 \%$ & $-0.6 \%$ \\
\hline 38. 39 & \pm 2 & 8 & 3 & 9.0 & 8.5764 & & & & \\
\hline 40,41 & \pm 4 & 8 & 2 & 9.0 & 8.6779 & & 8.6717 & $-2 \%$ & $-0.07 \%$ \\
\hline $\begin{array}{l}42 \\
43\end{array}$ & \pm 6 & 8 & 1 & 9.0 & $\begin{array}{l}8.8113 \\
8.8152\end{array}$ & 8.8084 & 8. 8112 & $-1 \%$ & $-0.02 \%$ \\
\hline 44,45 & \pm 8 & 8 & 0 & 9.0 & 9.0217 & 9.0151 & 9.0192 & $12 \%$ & $-0.03 \%$ \\
\hline 46,47 & \pm 1 & 9 & 4 & 10 & 9.444 & & & & \\
\hline $\begin{array}{l}48 \\
49\end{array}$ & \pm 3 & 9 & 3 & 10 & $\begin{array}{l}9.467 \\
9.552\end{array}$ & & & & \\
\hline 50,51 & \pm 5 & 9 & 2 & 10 & 9.629 & & 9.626 & $-0.8 \%$ & $-0.03 \%$ \\
\hline 52,53 & \pm 7 & 9 & 1 & 10 & 9.794 & & 9.794 & $0 \%$ & $0 \%$ \\
\hline $\begin{array}{l}54 \\
55\end{array}$ & \pm 9 & 9 & 0 & 10 & $\begin{array}{l}10.035 \\
10.036\end{array}$ & & 10.035 & $1 \%$ & $-0.005 \%$ \\
\hline 56 & 0 & 1.0 & 5 & 11 & 10.305 & & 10.221 & $-12 \%$ & $-0.8 \%$ \\
\hline 57,58 & \pm 2 & 10 & 4 & 11 & 10.318 & & & & \\
\hline 59,60 & \pm 4 & 10 & 3 & 11 & 10.463 & & 10.455 & $-1 \%$ & $-0.08 \%$ \\
\hline $\begin{array}{l}61 \\
62\end{array}$ & \pm 6 & 10 & 2 & 11 & $\begin{array}{l}10.573 \\
10.590\end{array}$ & & 10.581 & $-0.1 \%$ & $-0.005 \%$ \\
\hline 63,64 & \pm 8 & 10 & 1 & 11 & 10.774 & & 10.779 & $2 \%$ & $0.05 \%$ \\
\hline 65,66 & \pm 10 & 10 & 0 & 11 & 11.050 & & 11.053 & $-6 \%$ & $0.03 \%$ \\
\hline 67,68 & \pm 1 & 11 & 5 & 12 & 11.152 & & & & \\
\hline $\begin{array}{l}69 \\
70\end{array}$ & \pm 3 & 11 & 4 & 12 & $\begin{array}{l}11.160 \\
11.325\end{array}$ & & & & \\
\hline 71,72 & \pm 5 & 11 & 3 & 12 & 11.383 & & 11.383 & $0 \%$ & $0 \%$ \\
\hline
\end{tabular}


TABLE II (Continued)

\begin{tabular}{|c|c|c|c|c|c|c|c|c|c|}
\hline$n_{Q M}$ & $l$ & $n$ & $n_{r}$ & $E_{0}{ }^{\mathrm{a}}$ & $E_{Q N}{ }^{b}$ & $E_{M M}{ }^{\mathrm{c}}$ & $E_{B G N F}^{d}$ & $\Delta E_{r \in 1}{ }^{\theta}$ & $\Delta E_{\text {abs }}{ }^{\prime}$ \\
\hline 73,74 & \pm 7 & 11 & 2 & 12 & 11.534 & & 11.536 & $0.4 \%$ & $0.02 \%$ \\
\hline $\begin{array}{l}75 \\
76\end{array}$ & \pm 9 & 11 & 1 & 12 & $\begin{array}{l}11.750 \\
11.752\end{array}$ & & 11.764 & $5 \%$ & $0.11 \%$ \\
\hline 80,81 & \pm 11 & 11 & 0 & 12 & 12.065 & & 12.074 & $-12 \%$ & $-0.07 \%$ \\
\hline 77 & 0 & 12 & 6 & 13 & 11.966 & & 11. 864 & $-10 \%$ & $-0.9 \%$ \\
\hline 78,79 & \pm 2 & 12 & 5 & 13 & 11.968 & & & & \\
\hline 82,83 & \pm 4 & 12 & 4 & 13 & 12.206 & & & & \\
\hline $\begin{array}{l}84 \\
85\end{array}$ & \pm 6 & 12 & 3 & 13 & $\begin{array}{l}12.277 \\
12.334\end{array}$ & & 12.310 & $0.6 \%$ & $0.04 \%$ \\
\hline 86,87 & \pm 8 & 12 & 2 & 13 & 12.480 & & 12.491 & $2 \%$ & $0.09 \%$ \\
\hline 88,89 & \pm 10 & 12 & 1 & 13 & 12.712 & & 12.750 & $13 \%$ & $0.3 \%$ \\
\hline $\begin{array}{l}94 \\
97\end{array}$ & \pm 12 & 12 & 0 & 13 & $\begin{array}{l}13.077 \\
13.087\end{array}$ & & 13.097 & $-18 \%$ & $0.1 \%$ \\
\hline 91,92 & \pm 1 & 13 & 6 & 14 & 12.762 & & & & \\
\hline $\begin{array}{l}90 \\
93\end{array}$ & \pm 3 & 13 & 5 & 14 & $\begin{array}{l}12.748 \\
13.032\end{array}$ & & & & \\
\hline 95,96 & \pm 5 & 13 & 4 & 14 & 13.081 & & & & \\
\hline 98,99 & \pm 7 & 13 & 3 & 14 & 13.233 & & & & \\
\hline
\end{tabular}

${ }^{2}$ Energy of the uncoupled system. Calculated using present method including terms through $\Gamma^{(8)}$. ${ }^{\circ}$ Calculated by Don Noid. ${ }^{20} \Delta E_{\text {rel }}=\left(E_{B G N F}-E_{Q M}\right) / E_{0}-E_{Q M}$.

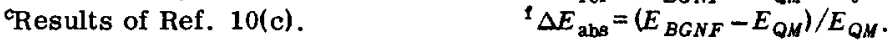

tify. It is possible that a higher degree calculation would give accurate energies for these states, but this is not certain, because the normal form is known to diverge in general. To identify these states, we arranged the quantum energy eigenvalues in a triangular matrix with $n$ and $l$ labeling the rows and columns. A pattern of energy gaps arises which persists up to the highest levels, and which, together with the pattern of degeneracies, serves to identify every state quite unambiguously. (The only two identifications that are even slightly uncertain are those of the nearly degenerate states 93 and 94 , but the identification given most accurately follows the pattern established by the other states.) Of the 56 states, or 29 "levels" in the region in which irregular classical motion appears, not one can definitely be said to fall outside the regular pattern.

\section{CONCLUSION}

We have presented a semiclassical method for approximating the energy eigenvalue spectrum of the Schrödinger equation for nonseparable systems of coupled oscillators. In this method, the classical problem is solved approximately by a sequence of canonical transformations which transform the Hamiltonian into the Birkhoff-Gustavson normal form. Two cases arise. Taking the zero-order solution to be that of a system of harmonic oscillators, we find that if the frequencies of the zero-order Hamiltonian are incommensurable, then the normal form can be written as a sum of products of one-dimensional harmonic oscillator Hamiltonians; if the frequencies of the zero-order Hamiltonian are commensurable, then additional terms which cannot be writ- ten as products of one-dimensional harmonic oscillator Hamiltonians enter into the normal form. The normal form in the incommensurable case can be quantized analytically to provide a simple formula for all the energy levels. In the commensurable case, one degree of freedom can be quantized anal ytically, and the other is quantized numerically by a one-dimensional phase integration. Consequently, a simple formula for the entire energy spectrum is not obtained, and each energy level must be generated individually; the numerical effort involved is typical of one-dimensional WKB methods, though somewhat complicated by the fact that the new Hamiltonian $\Gamma$ is not quadratic in the new momentum $J^{\prime}$.

The method has been applied to two two-dimensional cases, and it has been found to predict most of the energy levels accurately.

It is interesting to compare the method given here with two of the other presently available semiclassical methods for nonseparable systems. The present method is quite similar to the much older method of Born, ${ }^{24}$ but these methods differ in two ways: (i) the algebraic manipulations involved are different; (ii) in Born's method, terms in the expansion are collected in powers of a small parameter, while in the present method terms are collected in powers of the coordinates and momenta-the two collection schemes are not necessarily the same.

The methods of Marcus and his collaborators involve direct numerical integration of the classical trajectories. These methods give an essentially exact solution to the classical problem, but they have two principal 
limitations. They are generally more time consuming than the present method (which is extremely rapid and efficient), and for highly convoluted trajectories they can become difficult to apply. More important, however, these methods cannot be applied in regions of phase space in which irregular trajectories dominate, because for such trajectories, action variables cannot be defined. The present method cannot describe irregular motion, so it gives invariant tori, action variables, and regular energy levels even in regions in which the classical motion is irregular. It is a pleasant surprise that even these predicted energy levels are reasonably accurate-sufficiently accurate to establish the existence of a pattern to all of the quantum energy levels of the cases studied.

\section{ACKNOWLEDGMENTS}

We thank E. A. Remler for many productive conversations, Don Noid for generously performing many computations used in this research, and Carl Andersen for his assistance during the writing of the MACSYMA programs used for these calculations. The contributions of the Mathlab group at the Laboratory for Computer Science, MIT in making MACSYMA available are appreciated. ${ }^{25}$ This research was supported in part by grants from Research Corporation and from the $\mathrm{Na}-$ tional Science Foundation.

'H. Goldstein, Classical Mechanics (Addison-Wesley, Reading, Mass., 1965); L. A. Pars, A Treatise on Analytical Dynamics (Heinemann, London, 1965).

${ }^{2} \mathrm{~A}$. Wintner, Analytical Foundations of Celestial Mechanics (Princeton University, Princeton, 1947), p. 96.

${ }^{3}$ A. N. Kolomogorov, Dokl. Akad. Nauk. SSSR 98, 527 (1954); Proceedings of the International Congress of Mathematics, Noordhoff, Amsterdam, 1957, Vol. 1, p. 315.

'V. I. A rnol'd, Izv. Akad. Nauk. SSSR Ser. Mat. 25, 21 (1961); Usp. Mat. Nauk. 18, 81 (1963); 18, 13 (1963).

5(a) J. Moser, SIAM Review 8, 145 (1966); (b) J. Moser, Am. Math. Soc. Mem. 81, 1 (1968); (c) J. Moser, Stable and Random Motions in Dynamical Systems, Ann. Math. Studies (Princeton University, Princeton, 1973), Vol. 77; (d) J. Moser, Commum. Pure Appl. Math. 9, 673 (1956).

${ }^{6} \mathrm{~J}$. Ford, Fundamental Problems in Statistical Mechanics (North Holland, Amsterdam, 1975), Vol. 3, p. 215; also Advances in Chemical Physics, edited by I. Prigogine and S. A. Rice (Interscience, New York, 1973), Vol. 24, p. 155.

${ }^{7}$ The analogy is not perfect, because the irrationals form a dense set, while the set of regular trajectories is not necessarily dense in the set of all trajectories for a given system.

${ }^{8} \mathrm{M}$. Hénon and C. Heiles, Astron. J. 69, 73 (1964).
${ }^{9}$ (a) I. C. Percival, J. Phys. B 6, L229 (1973); (b) I. C. Percival, preprint; (c) N. Pomphrey, J. Phys. B 7, 1909 (1974); (d) see also A. Einstein, Verh. Dtsch. Phys. Ges. 19, 82 (1917).

${ }^{10}$ (a) W. Eastes and R. A. Marcus, J. Chem. Phys, 61, 4301 (1974); (b) D. W. Noid and R. A. Marcus, J. Chem. Phys. 62, 2119 (1975); (c) D. W. Noid and R. A. Marcus, J. Chem. Phys. 67, 559 (1977); (d) D. W. Noid, M. L. Koszykowski, and R. A. Marcus, J. Chem. Phys. 67, 404 (1977); (e) D. W. Noid, M. L. Koszykowski, R. A. Marcus, and J. D. McDonald, Chem. Phys. Lett. 51, 540 (1977).

${ }^{11}$ (a) I. C. Percival, J. Phys. A 7, 794 (1974); (b) I. C. Percival, N. Pomphrey, Mol. Phys. 31, 87 (1976).

${ }^{12}$ (a) S. Chapman, B. C. Garrett, and W. H. Miller, J. Chem. Phys. 64, 502 (1976); (b) W. H. Miller, J. Chem. Phys. 63, 996 (1975); (c) N. C. Handy, S. M. Colwell, and W. H. Miller, Discuss. Faraday Soc. 62, 29 (1976).

${ }^{13}$ (a) M. V. Berry and M. Tabor, Proc. R. Soc. (London) Ser. A 349, $101(1976)$; (b) K. S. Sorbie and N. C. Handy, Mol. Phys. 32, 1327 (1976); (c) K. S. Sorbie, Mol. Phys. 32, 1577 (1976).

${ }^{14}$ J. B. Delos and R. T. Swimm, Chem. Phys. Lett. 47, 76 (1977).

${ }^{15}$ R. T. Swimm, "Semiclassical Calculation of Vibrational Energy Levels for Nonseparable Systems," Ph. D. dissertation, College of William and Mary (1978). Preliminary accounts of this work were given by R. T. Swimm and J. B. Delos, Proceedings of the International Conference on Stochastic Behavior in Classical and Quantum Hamiltonian Systems, Como, 1977; and by J. B. Delos, Bull. Am. Phys. Soc. 23, 407 (1977).

${ }^{16}$ G. D. Birkhoff, Dynamical Systems (Am. Math. Soc., New York, 1966), Vol. IX.

${ }^{17}$ F. G. Gustavson, Astron. J. 71, 670 (1966)

${ }^{18}$ C. L. Siegel, Ann. Math. 42, 806 (1941); C. L. Siegel, Math. Ann. 128, 144 (1945); C. L. Seigel, Vorlesungen über Himmelsmechanik (Springer, Berlin, 1957).

${ }^{19}$ MACSYMA Reference Manual (The Mathlab Group, Laboratory for Computer Science, MIT, 1977 ${ }^{25}$; Proceedings of the 1977 MACSYMA Users' Conference (NASA CP-2012).

${ }^{20} \mathrm{D}$. W. Noid (private communication).

${ }^{21}$ J. B. Keller, Ann. Phys. (N.Y.) 4, 180 (1958).

${ }^{22}$ It also happens that, in the cases studied, the sign of the discrepancy between the eighth degree BGNF and the "exact" semiclassical energy levels is such that the BGNF result is often slightly closer to the exact quantum result, but we do not ascribe any special significance to this fact.

${ }^{23} \mathrm{Here}$ we are counting the nearly degenerate states with $l= \pm 3$, $\pm 6, \pm 9$ as one "level."

${ }^{24} \mathrm{M}$. Born, The Mechanics of the Atom (Ungar, New York, 1960).

${ }^{25}$ The Mathlab group at the Laboratory for Computer Science, MIT is supported, in part, by the United States Energy Research and Development Administration under Contract No. E(11-1)-3070 and by the National Aeronautics and Space Administration under Grant NSG 1323. 